\title{
Organocatalyzed synthesis of optically active cyanohydrins starting from alpha-ketoesters ${ }^{\dagger}$
}

\author{
Gonzalo de Gonzalo',** \\ 1 Departamento de Química Orgánica, Universidad de Sevilla, c/ Profesor García González 1, 41012, Sevilla, \\ Spain; gdegonzalo@us.es \\ * Correspondence: gdegonzalo@us.es; Tel.: +034-954-559-997 \\ + Presented at the 21 ${ }^{\text {st }}$ International Electronic Conference on Synthetic Organic Chemistry, 1-30 November \\ 2017.
}

Academic Editor: name

Received: date; Accepted: date; Published: date

\begin{abstract}
The synthesis of highly functionalized optically active cyanohydrins is described by performing the organocatalyzed selective addition of trimethylsilyl cyanide to a set of alpha-ketoesters employing different (thio)ureas as hydrogen bond catalysts. By optimizing certain parameters that affect to the organocatalytic process, as the temperature, solvent or cyanide concentration, it is possible to obtain the corresponding chiral cyanohydrins with excellent conversions and moderate to good optical purities.
\end{abstract}

Keywords: Organocatalysis; Thioureas; Chiral cyanohydrins; Process optimization

\section{Introduction}

Optically active cyanohydrins are very valuable compounds in organic chemistry, as these functionalized molecules can serve as synthons for a wide set of high value compounds, as $\alpha$-hydroxyketones, $\alpha$-hydroxyacids, $\alpha$-halonitriles or $\beta$-aminoalcohols, among others [1]. Different approaches have been developed for the preparation of chiral cyanohydrins, but the selective addition of a cyanide source to a carbonyl compound is the most common procedure. Several types of catalysts have been described for this selective process, including metal catalysts, biocatalysts or organocatalysts $[2,3]$.

The use of relatively small organic molecules is an area in constant development since the beginning of this century [4-6], and the use of hydrogen bond catalysts, in which the activation is performed by establishing a set of hydrogen bonds between the catalyst and the starting material, is a well-established technique in chemical synthesis [7,8]. Thus, different ureas and thioureas have been employed for the synthesis of chiral cyanohydrins, achieving in general the best results in presence of Jacobsen type (thio)ureas [9], or cinchona based catalysts [10]. Both types of hydrogen bond catalysts are bifunctional compounds, presenting in their structure a tertiary amine group, which is able to activate the cyanide nucleophile in addition to the electrophile activation debt to the (thio)urea moiety hydrogen bond formation [11]. The use of silyl cyanides as cyanation reagents has gained a great interest in the last few years, as these compounds present a good reactivity and they do not show the volatility and toxicity issues debt to hydrogen cyanide.

In the present proceeding, we are going to describe the use of different (thio)ureas for the preparation of chiral cyanohydrins derived from $\alpha$-ketoesters, with the aim of obtaining a set of highly functionalized chiral compounds.

\section{Methods}

\subsection{General procedure for the organocatalyzed cyanosilylation of ketoesters 1-5a in the optimized conditions}


The corresponding $\alpha$-ketoester 1-5a (0.6 mmoles) was dissolved in toluene $(1.0 \mathrm{~mL})$ containing $p$-nitrophenol (0.06 mmoles), catalyst VIb (0.06 mmoles, compound 5a) or catalyst VIIIb (0.06 mmoles, compounds 1-4a) and the system was cooled down to $-45^{\circ} \mathrm{C}$. After 10 minutes, trimethylsilylcyanide (1.5 equivalents) was added dropwise and the reactions were stirred at $-45^{\circ} \mathrm{C}$ for the times established. After this, a saturated solution of ammonium chloride $(2.0 \mathrm{~mL})$ was added to the crude reaction and the reaction was extracted with ethyl acetate $(2 \times 5.0 \mathrm{~mL})$. The organic phases were washed with brine $(5.0 \mathrm{~mL})$, dried onto sodium sulphate and the solvent was evaporated. The reactions were purified by column chromatography (toluene/EtOAc mixtures) to yield the corresponding $O$-silylated cyanohydrins (-)-1-5b with yields of $88 \%$ for $(-)-\mathbf{1 b}, 92 \%$ for $(-$ )-2b, $94 \%$ for (-)-3b, $90 \%$ for (-)-4b and $76 \%$ for $(-)-5 \mathbf{b}$.

\subsection{General method for the hydrolysis of O-silylated cyanohydrins (-)-1-5b.}

The corresponding $O$-silylated cyanohydrin $(-)-\mathbf{1 - 5} \mathbf{b}$ was dissolved in diethyl ether $(1.0 \mathrm{~mL})$ and at room temperature an aqueous solution of $\mathrm{HCl} 1.0 \mathrm{~N}(1.0 \mathrm{~mL})$ was added. After 2-3 hours it was observed the complete disappearance of the starting material (TLC in $n$-hexane/EtOAc mixtures). Once finished, the reaction was extracted with EtOAc $(2 \times 5 \mathrm{~mL})$, dried onto sodium sulphate and the solvent was evaporated under reduced pressure to yield the free cyanohydrins (-)-1-5c without further purification (yields higher than 90\%).

\section{Results and discussion}

Our initial studies were performed in the cyanosilylation of ethyl benzoylformate 1a in toluene employing 1.5 equivalents of trimethylsilyl cyanide (TMSCN) as mild cyanide source in presence of different (thio)ureas as organocatalysts (Scheme 1). The obtained $O$-silylcyanohydrins can be easily transformed into the free cyanohydrins by mild treatment with a diluted aqueous solution of $\mathrm{HCl}$ $(1.0 \mathrm{~N})$ and THF, obtaining in all cases quantitative yields. Initially, we have tested the sugar-based thioureas I-II, which have been prepared in our research group and employed in the selective addition of formaldehyde tert-butylhydrazone to aliphatic $\alpha$-ketoesters (entries 1 and 2, Table 1) [12]. After long times, low conversions were obtained while the final product was obtained almost racemic. The use of an indanol-based thiourea as III also led to a poor cyanosilylation reaction. Only $8 \%$ of $(-)-\mathbf{1 b}$ was recovered after 70 hours at $-15^{\circ} \mathrm{C}$.

The BINAM-derived bis-urea IV, that has been also successfully employed in the formaldehyde tert-butylhydrazone addition to aromatic $\alpha$-ketoesters [13], did not catalyze the cyanosilylation at $-30^{\circ} \mathrm{C}$ even after 72 hours. By this reason, we have prepared a BINAM-monourea containing a tertiary amine in its structure (catalyst Va) [14]. The cyanosilylation catalyzed by this compound afforded the $O$-silylated cyanohydrin with $78 \%$ conversion after 40 hours, and a modest selectivity (entry 5). Further $\mathrm{N}$-methylation of $\mathbf{V a}$ and treatment with a cyanide salt afforded the quaternary ammonium-thiourea $\mathbf{V b}$. The use of this catalyst allows a slight increase in both the activity and selectivity, as shown in entry 6 , being possible to achieve (-)-1b with $86 \%$ conversion and $25 \%$ ee. 


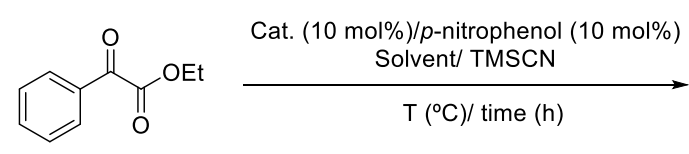

1a

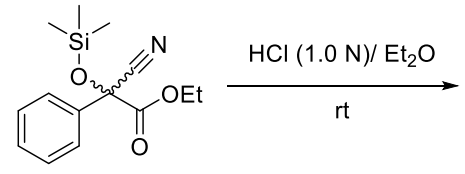

$(-)-1 b$

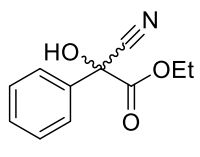

$(-)-1 c$<smiles>CNNC(=S)NC1C(O)C(O)OC(CO)C1O</smiles>

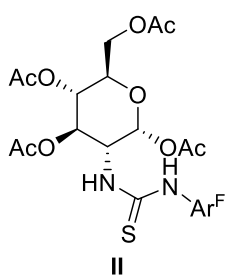<smiles>OC1c2ccccc2CC1NC(=S)N[I+]</smiles>

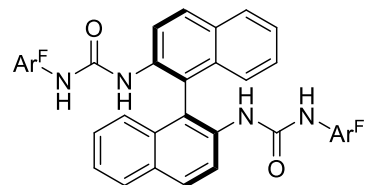

IV<smiles>CN(C)c1ccc2ccccc2c1-c1c(NC(=O)N[18F])ccc2ccccc12</smiles>

III<smiles>CCN1C2CCN(C2)C1C(O)c1ccnc2ccc(OC)cc12</smiles>

VIIa

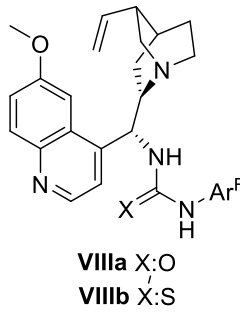

Scheme 1. Cyanosilylation of ethyl benzoylformate 1a catalyzed by a set of (thio)ureas. Ar ${ }^{\mathrm{F}}$ refers to the bis-3,5-trifluoromethylphenyl group.

Table 1. Organocatalyzed cyanosilylation of prochiral ethyl benzoylformate (1a) in toluene. ${ }^{1}$

\begin{tabular}{cccccc}
\hline Entry & Catalyst & T ( $\left.{ }^{\circ} \mathbf{C}\right)$ & time $(\mathbf{h})$ & Conversion $\mathbf{~ ( \% )}^{\mathbf{2}}$ & $\boldsymbol{e e}(\mathbf{~} \%)^{\mathbf{3}}$ \\
\hline 1 & I & -15 & 72 & 13 & $\leq 3$ \\
2 & II & -15 & 48 & 27 & 8 \\
3 & III & -15 & 70 & 8 & n.d. \\
4 & IV & -30 & 72 & $\leq 3$ & n.d. \\
5 & Va & -45 & 40 & 78 & 17 \\
6 & Vb & -45 & 24 & 86 & 25 \\
7 & VIa & -45 & 24 & 80 & 41 \\
8 & VIb & -45 & 40 & 83 & 27 \\
9 & VII & -45 & 24 & 92 & 5 \\
10 & VIIIa & -45 & 24 & $\geq 97$ & 48 \\
11 & VIIIb & -45 & 24 & 86 & 63 \\
\hline
\end{tabular}

${ }^{1}$ For reaction details, see Experimental Section. ${ }^{2}$ Determined by ${ }^{1} \mathrm{H}-\mathrm{NMR}$ in $\mathrm{CDCl}_{3} .{ }^{3}$

Determined by HPLC after hydrolysis to the free cyanohydrin (-)-1c. n.d. not determined.

In view of these results, we decided to test two Jacobsen type thioureas (VIa-b), which have been previously employed in cyanosilylation processes. As shown in entry 7, the thiourea presenting an imine moiety in its structure allowed to obtain (-)-1b with $41 \%$ ee and a conversion of $80 \%$ after 24 hours. When the thiourea contains a primary amine (VIb, entry 8), the optical purity of the cyanohydrin decayed to $25 \%$ while the reaction was slower (83\% conversion after $40 \mathrm{~h}$ ). Quinine was able to perform the cyanosilylation of $\alpha$-ketoester 1a with a 92\% conversion after 24 hours, but without selectivity (entry 9). Thus, we have prepared both a cinchona-based urea (VIIIa) and thiourea (VIIIb) containing a 3,5-bistrifluoromethylphenyl group in order to increase the system reactivity [15]. Both catalysts were able to catalyze the cyanosilylation of 1a with good performance in toluene at $-45^{\circ} \mathrm{C}$. Thus, the use of urea VIIIa afforded (-)-1b with complete conversion and $48 \%$ ee after 24 hours, while the reaction in presence of the thiourea VIIIb was slower $(86 \%$ conversion in 
the same time) but showed the highest enantioselectivity ( $63 \%$ ee). Once selected the cinchona-based (thio)ureas VIIIa-b as the most suitable organocatalysts for the cyanosilylation of substrate 1a, we have studied some of the reaction parameters than can affect to the process activity and selectivity. Initially, the reaction catalyzed by urea VIIIa at $-45^{\circ} \mathrm{C}$ has been tested in different organic solvents, as shown in Table 2. Conversions higher than $90 \%$ can be achieved using tert-butyl methyl ether (entry 2) or dichloromethane (entry 3), while the use of diethyl ether and hexane afforded lower activities. For all the solvents tested, much lower selectivities were obtained compared to toluene.

Table 2. Solvent effect in the synthesis of cyanohydrin (-)-1b catalyzed by urea VIIIa at $-45^{\circ} \mathrm{C}$ after 24 hours.

\begin{tabular}{cccc}
\hline Entry & Solvent & Conversion $\mathbf{( \% )}$ & $\boldsymbol{e e} \mathbf{( \% )} \mathbf{~}^{\mathbf{1}}$ \\
\hline 1 & Toluene & $\geq 97$ & 48 \\
2 & tert-Butyl methyl ether & 90 & 29 \\
3 & Dichloromethane & 96 & 26 \\
4 & Diethyl ether & 83 & 36 \\
5 & n-Hexane & 78 & 33 \\
\hline
\end{tabular}

${ }^{1}$ Determined by ${ }^{1} \mathrm{H}-\mathrm{NMR}$ in $\mathrm{CDCl}_{3} .{ }^{2}$ Determined by HPLC after hydrolysis to the free cyanohydrin (-)-1c.

The effect of the temperature in the organocatalyzed addition of TMSCN has been also studied in the VIIIa-organocatalyzed addition, as shown in Figure 1. As the reactions were stopped at different times for each temperature, we have defined the reaction rate as the mmoles of substrate 1a consumed per hour and per $\mathrm{mL}$, in order to compare the conversions obtained. As expected, the increase of the temperature led to an important increase in the reaction rate, from $0.7 \mathrm{mmol} \mathrm{mL}^{-1} \mathrm{~h}^{-1}$ at $-78^{\circ} \mathrm{C}$ up to $4.9 \mathrm{mmol} \mathrm{mL}^{-1} \mathrm{~h}^{-1}$ at $-15^{\circ} \mathrm{C}$, but also to a high decrease in the system selectivity, especially at temperatures higher than $-45^{\circ} \mathrm{C}$, as the optical purity of $(-)-1 \mathbf{b}$ passed from $48 \%$ at $-45^{\circ} \mathrm{C}$ to $21 \%$ at $-30^{\circ} \mathrm{C}$.

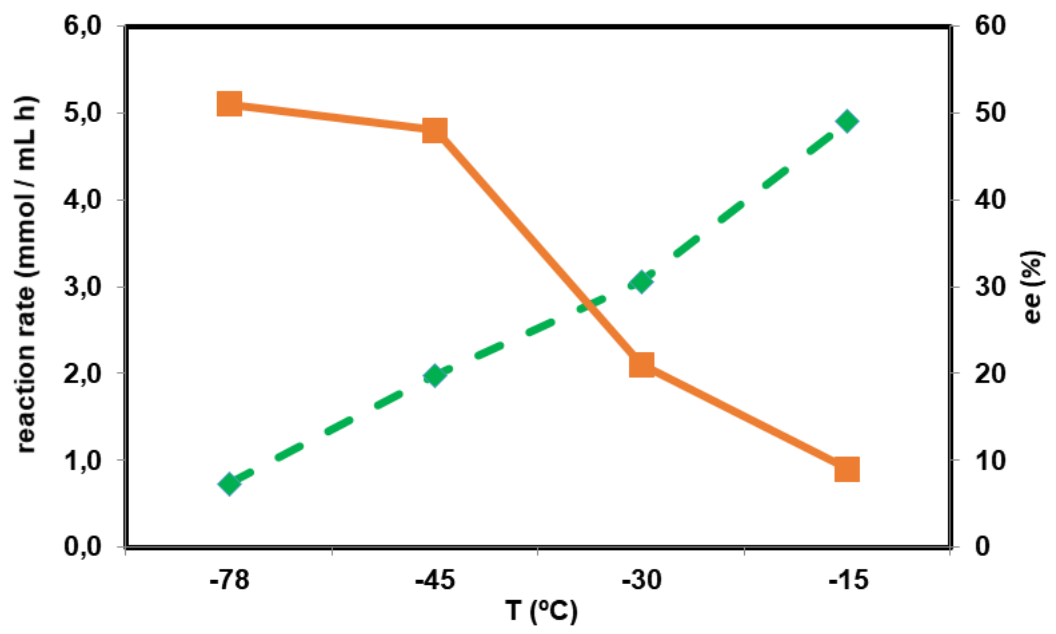

Figure 1. Effect of the temperature in the activity (dashed green line) and the selectivity (continuous red line) of the cyanosilylation of ethyl benzoylformate 1a catalyzed by thiourea VIIIb in toluene.

Once selected this temperature as the best for the process, we have analyzed the influence of the cyanide source concentration in the reaction, as shown in Table 3. The use of higher amounts of cyanide afforded slightly higher reaction rates but also led to an important decrease in the system selectivity. Thus, when the cyanosilylation was performed with 5.0 equivalents of cyanide (entry 4 ) $(-)-\mathbf{1 b}$ was recovered with only $13 \%$ ee. The use of 1.0 equivalent of cyanide does not increase the optical purity of the final product, but the reaction rate suffers an important decrease, as shown in entry 1 . It can be established that 1.5 equivalents results to be a proper amount for this process. 
Table 3. Effect of the trimethylsilyl cyanide in the cyanosilylation of 1a catalyzed by thiourea VIIIa at $-45^{\circ} \mathrm{C}$ after 24 hours. $^{1}$

\begin{tabular}{cccc}
\hline Entry & Cyanide equivalents & Rate $\left(\mathbf{m m o l ~ L}^{-\mathbf{1}} \mathbf{h}^{-1}\right)$ & ee $(\%)^{\mathbf{2}}$ \\
\hline 1 & 1.0 & 12.3 & 47 \\
2 & 2.0 & 48.4 & 48 \\
3 & 2.0 & 54.6 & 43 \\
4 & 5.0 & 57.2 & 13 \\
\hline
\end{tabular}

${ }^{2}$ Determined by ${ }^{1} \mathrm{H}-\mathrm{NMR}$ in $\mathrm{CDCl}_{3} .{ }^{3}$ Determined by HPLC after hydrolysis to the free cyanohydrin (-)-1c.

Acetone cyanohydrin has been also employed as cyanide source instead of trimethylsilyl cyanide. The use of this reagent allows obtaining directly the free cyanohydrin without requiring a hydrolysis step. Unfortunately, the reaction of $\mathbf{1 a}$ in toluene catalyzed by thiourea VIIIb afforded $85 \%$ conversion after 36 hours, while $(-)-\mathbf{1 b}$ was obtained with only $37 \%$ ee.

It has been described that the presence of certain additives as alcohols and phenols in organocatalyzed cyanosilylation has a positive effect in both the activity and selectivity of the reactions, as these additives can improve the formation of the cyanide nucleophile in the reaction medium. By this reason, we have studied the use of three alcohols in $10 \mathrm{~mol} \%$ extent in the VIIIb-catalyzed cyanosilylation of ethyl benzoylformate 1a. As can be observed in Table 4, all the three alcohols increased the systems activity, being possible to recover $(-)-\mathbf{1 b}$ with conversions higher than $90 \%$ after 24 hours. Regarding the selectivity, the addition of $10 \mathrm{~mol} \%$ of $p$-nitrophenol allows to increase the process selectivity, as the chiral cyanohydrin was obtained with $68 \% e e$, while for the aliphatic alcohols, the final product is recovered with slightly lower selectivity.

Table 4. Cyanosilylation of ketoester 1a catalyzed by VIIIb in presence of different alcohols as additives at $-45^{\circ} \mathrm{C}$ after 24 hours.

\begin{tabular}{cccc}
\hline Entry & Additive & Conversion $\mathbf{( \% )}$ & $\boldsymbol{e e}(\mathbf{\%})^{\mathbf{2}}$ \\
\hline 1 & None & 86 & 63 \\
2 & 2,2,2-trifluoroethanol & 93 & 56 \\
3 & tert-butanol & 92 & 61 \\
4 & $p$-nitrophenol & 95 & 68 \\
\hline
\end{tabular}

${ }^{1}$ Determined by ${ }^{1} \mathrm{H}-\mathrm{NMR}$ in $\mathrm{CDCl}_{3} .{ }^{2}$ Determined by HPLC after hydrolysis to the free cyanohydrin (-)-1c.

In order to explore the substrate profile of this organocatalyzed process, the best conditions found for compound 1a (toluene, $-45^{\circ} \mathrm{C}$ and $10 \mathrm{~mol} \%$ thiourea VIIIb and $10 \mathrm{~mol} \%$ of $p$-nitrophenol) were then extended to other aromatic $\alpha$-ketoesters, as shown in Scheme 2. Thus, methyl benzoylformate reacted with TMSCN leading to (-)-2b with $92 \%$ conversion and $56 \%$ ee, slightly lower than the achieved for the ethyl analogue. The cyanosilylation of ethyl 4-cyanobenzoylformate 3a occurred with $93 \%$ conversion after 24 hours, recovering (-)-3b with $62 \%$ ee. A heteroaromatic $\alpha$-ketoester as $4 \mathbf{a}$ was also converted in the (-)-O-silylcyanohydrin, but with a lower conversion and optical purity $(55 \%$ ee) than its aromatic analogue $1 \mathbf{a}$. 


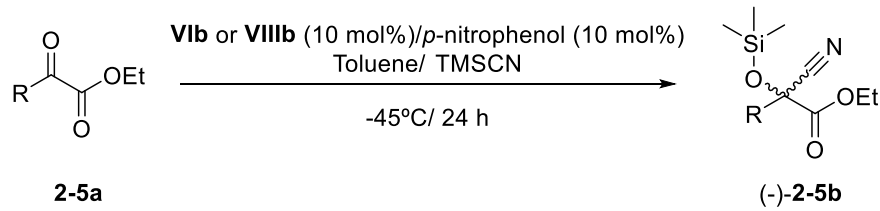

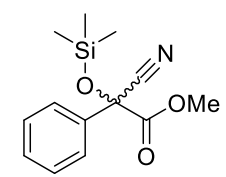

$(-)-\mathbf{2 b}$, VIII b $92 \%, 56 \%$ ee

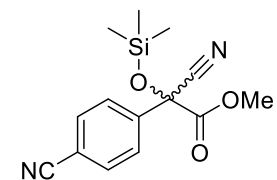

$(-)-3 \mathbf{b}$, VIIIlb $93 \%, 62 \%$ ee

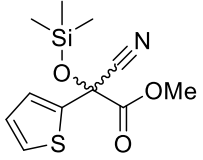

(-)-3b, VIIIb $78 \%, 55 \%$ ee

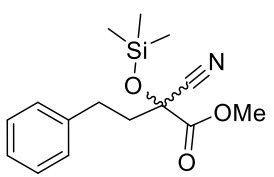

$(-)-5 \mathbf{b}$, Vlb $99 \%, 47 \%$ ee

Scheme 2. Substrate scope in the organocatalyzed cyanosilylation of $\alpha$-ketoesters employing thioureas VIb and VIIIb.

The cyanosilylation of ethyl 4-phenyloxobutyrate (5a) in presence of the cinchona-based thiourea VIIIb afforded the $O$-silyl cyanohydrin with a good activity but with a low selectivity ( $33 \%$ $e e$ ). This $\alpha$-ketoester is not an aromatic one, as the reactive center is linked to an aliphatic carbon atom, which can explain its different behavior. We have tested other catalysts in its cyanosilylation, achieving the best results in presence of the Jacobsen thiourea VIb. In these conditions, (-)-5b was recovered with complete conversion and a $49 \%$ ee after 24 hours.

\section{Conclusions}

The cyanosilylation of a set of prochiral $\alpha$-ketoesters catalyzed by different (thio)ureas allows obtaining the corresponding chiral $\mathrm{O}$-silyl cyanohydrins presenting an ester moiety with high conversions and moderate optical purities. Different parameters that can affect to the organocatalyzed process have been optimized (temperature, cyanide source and its concentration, solvent, additives), in order to achieved the highest optical purities. Thus, best conditions for the cyanosilylation of aromatic substrates were achieved with a cinchona-based thiourea in toluene at $-45^{\circ} \mathrm{C}$ in presence of $10 \mathrm{~mol} \%$ of $p$-nitrophenol, while for the aliphatic substrate the highest selectivity was obtained with a Jacobsen type thiourea. This process represents the first step to obtain chiral functionalized cyanohydrins, valuable synthons in organic synthesis.

Acknowledgments: G.d.G. thanks MINECO (Ramón y Cajal Program) for personal funding.

Author Contributions: G.d.G performed the experiments, conceived, designed and wrote the paper.

Conflicts of Interest: The author declares no conflict of interest.

\section{References}

1. Gregory, R. J. H. Cyanohydrins in nature and the laboratory: Biology, preparations, and synthetic applications. Chem. Rev. 1999, 99, 3649-3682. DOI: 10.1021/cr9902906

2. Kurono, N.; Ohkuma, T. Catalytic asymmetric cyanation reactions. ACS Catal. 2016, 6, 989-1023. DOI: 10.1021/acscatal.5b02184.

3. North, M.; Usanov, D. L.; Young, C. Lewis acid catalyzed asymmetric cyanohydrin synthesis. Chem. Rev. 2008, 108, 5146-5226. DOI: $10.1021 / \mathrm{cr} 800255 \mathrm{k}$

4. Dalko, P. I. Comprenhensive Enantioselective Organocatalysis: Catalysts, Reactions and Applications, 1st ed.; Wiley-VCH, Weinheim, Germany, 2013. ISBN: 978-3-527-33236-6

5. Grondal, C.; Jeanty, M.; Enders, D. Organocatalytic cascade reactions as a new tool in organic synthesis. Nature Chem. 2010, 2, 167-178. DOI: 10.1038/nchem.539

6. List, B. Asymmetric Organocatalysis, 1st ed.; Springer-Verlag, Berlin, Germany, 2010. ISBN: 978-3-642-02814-4 
7. Kotke, M.; Schreiner, P. R. (Thio)urea organocatalysts. In Hydrogen bonding in organic synthesis, $1^{\text {st }}$ ed.; Pihko, P. M.; Wiley-VCH, Weinheim, Germany, 2009, pp. 141-352. ISBN: 978-3-527-31895-7.

8. Zhang, Z.; Schreiner, P. R. (Thio)urea organocatalysis-What can be learnt from anion recognition?. Chem. Soc. Rev. 2009, 38, 1187-1198. DOI: 10.1039/b801793j

9. Zuend, S. J.; Jacobsen, E. N. Cooperative catalysis by tertiary amino-thioureas: Mechanism and basis for enantioselectivity of ketone cyanosilylation. J. Am. Chem. Soc. 2007, 129, 15872-15883. DOI: 10.1021/ja0735352

10. Kong, S.; Fan, W.; Wu, G.; Miao, Z. Enantioselective synthesis of tertiary $\alpha$-hydroxy phosphonates catalyzed by carbohydrate/cinchona alkaloid thiourea organocatalysts. Angew. Chem. Int. Ed., 2012, 51, 8864-8867. DOI: 10.1002/anie.201204287.

11. Fuerst, D. E.; Jacobsen, E. N. Thiourea catalyzed enantioselective cyanosilylation of ketones. J. Am. Chem. Soc. 2005, 127, 8964-8965. DOI: 10.1021/ja052511x.

12. Carmona, J. A.; de Gonzalo, G.; Serrano, I.; Crespo-Peña, A. M.; Šimek, M.; Monge, D.; Fernández, R.; Lassaletta, J. M. Asymmetric organocatalytic synthesis of tertiary azomethyl alcohols: key intermediates towards azoxy compounds and $\alpha$-hydroxy- $\beta$-amino esters. Org. Biomol. Chem. 2017, 15, 2993-3005. DOI: 10.1039/c7ob00308k

13. Crespo-Peña, A. M.; Monge, D.; Martín-Zamora, E.; Álvarez, E.; Fernández, R.; Lassaletta, J. M. Asymmetric formal carbonyl-ene reactions of formaldehyde tert-butyl hydrazone with $\alpha$-keto esters: Dual activation by Bis-urea catalysts. J. Am. Chem. Soc. 2012, 134, 12912-12915. DOI: 10.1021/ja305209w.

14. Bernal, P.; Fernández, R.; Lassaletta, J.M. Organocatalytic asymmetric cyanosilylation of nitroalkenes. Chem. Eur. J. 2010, 16, 7714-7718. DOI: 10.1002/chem.201001107.

15. Jakab, G.; Tancon, C.; Zhang, Z.; Lippert, K. M.; Schreiner, P. R. (Thio)urea organocatalyst equilibrium acidities in DMSO. Org. Lett. 2012, 14, 1724-1727. DOI: 10.1021/ol300307c.

(C) 2017 by the authors. Submitted for possible open access publication under the terms and conditions of the Creative Commons Attribution (CC BY) license (http://creativecommons.org/licenses/by/4.0/). 